\title{
Relação entre desenvolvimento motor corporal e aquisição de habilidades orais $* * *$
}

\author{
Relationship between the motor development of the body and the \\ acquisition of oral skills
}

\author{
Mariângela Silva Telles* \\ Célia Sperandeo Macedo**
}

*Fonoaudióloga. Mestre em Pediatria na Faculdade de Medicina Botucatu Universidade Estadual Paulista (Unesp). Endereço para

correspondência:

Rua Saint Martin, 19-60 - Bauru - São Paulo - CEP 17.012-080

(mariangelatelles@hotmail.com)

**Médica. Livre Docente. Pediatra do Departamento de Pediatria da Faculdade de Medicina de Botucatu Unesp.

***Trabalho Realizado na Faculdade de Medicina de Botucatu - Unesp.

Artigo Original de Pesquisa

Artigo Submetido a Avaliação por Pares

Conflito de Interesse: não

Recebido em 04.06.2007. Revisado em 01.04.2008; 25.04.2008; 26.05.2008.

Aceito para Publicação em 26.05.2008.

\begin{abstract}
Background: the literature points to the influence of body posture on the oral skills of children which sensorimotor deficits. Only a few studies with normal children exist on this subject. Aim: to study the relationship between motor skills and oral motor skills in children, from the first day of life to 24 months of age. Method: 42 children were video recorded at the first day of life, and at 1, 2, 3, 4, 5, 6, 9, 12 and 24 months of age. Recordings were made in the following postures: supine, prone, seated, standing and during breast and bottle feeding (until 5 months), using spoon (purée: 3 - 12 months); cup (water or juice: 6 - 24months) and eating solid food (6 -24 months). Quantitative scores for body motor development and oral skills were established; and for the statistical analysis the Pearson Correlation Coefficient Test was used with a significance level of 5\%. Results: the results of motor development point to similar data between supine, prone, seated and standing positions; for the oral motor skills (during feeding/ breastfeeding, using spoon, cup and chewing). A similarity was observed in the acquisition of motor abilities related to the lips, tongue and jaw in each of the feeding situations. There was an association between the motor and the oral motor skills; the results indicate that the motor development (motor skills) occurred prior to the development of the oral skills from the 5th to 24 months and that the skills related to the jaw when using a cup and spoon occurred prior to the development of the skills related to the lips and tongue. Conclusion: there was a growing increase in the acquisition of motor and oral skills along the ages, as well as a variability of skills in the ages between the 3rd and 24 months and a significant association between the motor and oral skills.
\end{abstract}

Key Words: Stomatognathic System; Children; Feeding; Oral Motor Development.

\section{Resumo}

Tema: a literatura aponta para a influência da postura corporal sobre as habilidades orais em crianças com desenvolvimento sensório-motor alterado. Em crianças normais existem poucos estudos sobre essa relação. Objetivo: estudar em crianças a termo a relação entre habilidades motoras e habilidades motoras orais, desde 1 dia de vida até 24 meses de idade. Método: 42 crianças foram filmadas com 1 dia, 1 mês, 2 , 3, 4, 5, 6, 9,12 e 24 meses nas posições supino, prono, sentado e em pé e durante alimentação com amamentação / mamadeira (até 5 meses), uso de colher para alimentação pastosa (3 aos 12 meses), uso de copo para água ou suco ( 6 aos 24 meses) e alimento sólido ( 6 aos 24 meses). Estabeleceram-se escores de quantificação para o desenvolvimento corporal e habilidades orais e utilizou-se o coeficiente de correlação de Pearson para o estudo estatístico, adotando-se nível de significância de 5\%. Resultados: os resultados do desenvolvimento motor apontaram para semelhança de dados entre supino e prono e sentado e em pé; para as habilidades orais (durante a alimentação com mamadeira/amamentação, colher, copo, mastigação) constatou-se em cada modalidade de alimentação, homogeneidade de aquisição de habilidades para lábios, língua e mandíbula. Houve associação entre habilidades motoras e orais; resultados apontam que o desenvolvimento motor (habilidades motoras) se deu antes das orais desde o $5^{\circ}$ ao $24^{\circ}$ mês e que as habilidades de mandíbula em copo e colher ocorreram antes das habilidades de lábios e língua. Conclusão: houve crescente aquisição de habilidades motoras e orais, variabilidade de habilidades em idades entre 3 e 24 meses e associação entre habilidades motoras e orais.

Palavras-Chave: Sistema Estomatognático; Criança; Alimentação; Desenvolvimento Motor Oral.

Referenciar este material como: 


\section{Introdução}

A maturação das habilidades motoras e habilidades para a alimentação são alcançadas concomitantemente ao desenvolvimento do sistema nervoso central (SNC) associada à experiência de aprendizado $^{(1-2)}$. No primeiro ano de vida, existe estreita relação entre a evolução do SNC e funções que aparecem ou desaparecem, funções reflexas evoluem para complexas e voluntárias; algumas atividades presentes ao nascimento são "inibidas" no primeiro ano, ressurgindo como atividades voluntárias e complexas automatizado-se em nível superior no sistema nervoso ${ }^{(3)}$.

O desenvolvimento motor evolui de forma organizada sendo cada etapa conseqüência da precedente e necessária à posterior ${ }^{(4)}$. Nos primeiros dois anos padrões progressivos de desenvolvimento motor global e fino e motor oral são pré-requisitos para habilidades de auto alimentação favorecendo o estado nutricional e o crescimento de lactentes ${ }^{(5)}$.

Após o quarto ou quinto mês de vida, a condição reflexa é substituída pela movimentação oral voluntária para alimentação e para a fala ${ }^{(6-8)}$, tornado-se mais efetiva com a estabilidade da cabeça, melhorando o controle da mandíbula que é influenciado pelo alinhamento do tronco o qual depende da estabilidade da área pélvica ${ }^{(9-10)}$.

Desde 1990 sugiram escalas para acompanhamento longitudinal de crianças que consistem na observação e classificação dos movimentos espontâneos considerando freqüência, amplitude e força do movimento, portanto sua complexidade e variabilidade. A escala motora Alberta Infant Motor Scale ${ }^{(11)}$ avalia crianças nas posições prono, supina, sentada, em pé e descarga de peso. Similarmente, Green e Pountney ${ }^{(12)}$ desenvolveram medida dos Níveis de Habilidades de Chailey. Assim as avaliações do comportamento motor devem permitir quantificar e qualificar pequenas mudanças de habilidades nos primeiros meses de vida mostrando a evolução do controle postural e a seqüência de aquisições no transcorrer do estudo evolutivo ${ }^{(11)}$

Pesquisas demonstraram existir associação entre controle postural e sistema estomatognático, e influência recíproca da postura corporal sobre as estruturas orais ${ }^{(13-15)}$; crianças com desordens sensório motoras necessitam de adequado controle para êxito na alimentação ${ }^{(11,16-17)}$.

Estudos sobre a função oral na alimentação em crianças normais ${ }^{(8,18-22)}$ trouxeram importantes avanços, mas poucos estudos abordando relações entre sistema motor global e habilidades motoras orais em crianças normais foram realizados.

Propôs-se neste estudo descrever a relação entre desenvolvimento motor corporal e aquisição de habilidades orais em crianças normais de 1 dia até 24 meses.

\section{Método}

Pesquisa observacional - longitudinal; estudouse 42 crianças, 26 meninos e 16 meninas. O estudo foi aprovado pelo Comitê de Ética em Pesquisa da Faculdade de Medicina de Botucatu - UNESP (OF. 179/2002 - CEP MJBV/asc), e o termo livre e esclarecido foi assinado pelos responsáveis pelos bebês.

Foram incluídos no estudo recém nascidos a termo, peso adequado para idade gestacional (23), apgar 9 e 10 no quinto minuto, ausência de másformações, infecções congênitas, alterações anatômicas orofaciais, alimentados por via oral e concepto único.

As crianças foram avaliadas e filmadas em média 15 minutos no primeiro dia de vida, com 1, 2, 3, 4, 5, $6,9,12$ e 24 meses. Filme de 10 crianças ( $25 \%$ ) foram examinados por segundo observador para determinação da confiabilidade.

As variáveis estudadas foram habilidades motoras e habilidades orais, definindo-se como habilidade motora à resposta corporal nas posições: supino, prono, sentado, em pé. Cada criança foi colocada sobre mesa de vidro temperado, inspirada no modelo do Centro de Chailey Heritage da Inglaterra ${ }^{(12)}$, aquecida a $29^{\circ} \mathrm{C}$, com espelho inclinado a $45^{\circ}$, para visualização dos pontos de apoio, posicionada em: supino, prono, sentada, em pé até 4 meses; sentada sem apoio aos 6, 9, 12 meses e deambulação sobre a mesa e solo aos 9, 12 e 24 meses.

As habilidades orais foram definidas como a resposta motora oral durante alimentação com amamentação/mamadeira, oferta de alimento pastoso em colher de metal, alimento sólido e líquido em copo de $120 \mathrm{ml}$.

Foi oferecida alimentação na seguinte sequiência:

. até 2 meses, amamentação ou mamadeira; dedo enluvado introduzido na cavidade oral para verificação dos movimentos de língua antes da oferta;

. 3 aos 5 meses introduziu-se papa de fruta em quantidade padronizada $(3 \mathrm{ml})$;

. 6 meses papa de fruta $(5 \mathrm{ml})$, bolacha de textura 
macia na mão da criança e após a deglutição, introduziu-se porção de bolacha na parte vestibular oral para observação da lateralização da língua, e suco ou água em copo;

.9 meses, acrescentou-se bolacha de textura dura na mão da criança;

.12 e 24 meses, bala de gelatina.

Adotou-se para avaliação das habilidades motoras seis níveis de habilidades na posição supino e prono e sete na posição sentado descritos por Green et al. ${ }^{(12)}$ desde recém nascido até onze meses. Para posição em pé até doze meses adotouse a descrição de Bly ${ }^{(24)}$; e para aos vinte e quatro meses a descrição de Nardi e Porto ${ }^{(25)}$, elaborou-se sete níveis baseados para essa posição.

Os níveis foram estabelecidos para observação das atividades espontâneas como, movimentos da cabeça, cintura escapular, tronco, pélvis, membros, descarga de peso e habilidade para movimentar-se fora das posições inicialmente colocados.

A partir das descrições de Morris e Klein ${ }^{(26)}$ elaborou-se níveis de habilidades do sistema motor oral, considerando-se três níveis para amamentação ou mamadeira, quatro para uso de colher e copo e cinco para mastigação. Em cada nível e modalidade avaliou-se a posição e os movimentos peculiares da língua, lábios e mandíbula.

Cada nível foi considerado mediante a presença de $70 \%$ das características.

Os dados coletados foram classificados através de análise descritiva, quantitativa e quantitativa e estatística descritiva para estudo das variáveis motoras e orais, utilizou-se coeficiente de correlação linear de Pearson para estudo da associação entre as variáveis e cálculo das porcentagens dos níveis. $\mathrm{O}$ teste de Wilcoxon para análise de confiabilidade; considerou-se nível de significância de $5 \%$.

\section{Resultados}

A concordância entre os examinadores foi de $86,7 \%$.

Observou-se nas habilidades motoras e motoras orais homogeneidade das médias e medianas em supino, prono e sentado e em pé, e de língua, lábios e mandíbula em amamentação/mamadeira, uso de colher, copo e na mastigação obtidas pela somatória da pontuação dos níveis.

Houve associações significativas entre as variáveis das habilidades motoras e orais (Tabela 1).

TABELA 1. Medida de associação entre variáveis motoras e orais.

\begin{tabular}{|c|c|c|c|c|}
\hline & Variáveis Orais & Variáveis Motoras & Valor de $\mathrm{r}$ & Valor de $\mathrm{p}$ \\
\hline \multirow{9}{*}{ 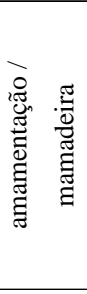 } & língua & supino & 0,4244 & 0,0050 \\
\hline & língua & prono & 0,4732 & 0,0016 \\
\hline & língua & sentado & 0,4183 & 0,0058 \\
\hline & língua & em pé & 0,4530 & 0,0013 \\
\hline & lábios & sentado & 0,4058 & 0,0076 \\
\hline & mandíbula & supino & 0,4558 & 0,0024 \\
\hline & mandíbula & prono & 0,4496 & 0,0028 \\
\hline & mandíbula & sentado & 0,3071 & 0,0480 \\
\hline & mandíbula & em pé & 0,4444 & 0,0032 \\
\hline \multirow{7}{*}{$\frac{\bar{\Xi}}{\bar{D}}$} & língua & supino & 0,4097 & 0,0070 \\
\hline & língua & prono & 0,3211 & 0,0382 \\
\hline & língua & sentado & 0,3535 & 0,0216 \\
\hline & lábios & supino & 0,3079 & 0,0472 \\
\hline & lábios & em pé & 0,3222 & 0,0374 \\
\hline & mandíbula & supino & 0,4679 & 0,0018 \\
\hline & mandíbula & prono & 0,3202 & 0,0386 \\
\hline \multirow{5}{*}{ ঠి } & língua & supino & 0,3678 & 0,0166 \\
\hline & língua & em pé & 0,4609 & 0,0011 \\
\hline & lábios & supino & 0,3236 & 0,0366 \\
\hline & lábios & em pé & 0,3565 & 0,0206 \\
\hline & mandíbula & em pé & 0,2940 & 0,0480 \\
\hline \multirow{7}{*}{ 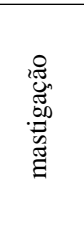 } & língua & supino & 0,4018 & 0,0020 \\
\hline & lábios & supino & 0,4480 & 0,0030 \\
\hline & lábios & em pé & 0,4214 & 0,0054 \\
\hline & mandíbula & supino & 0,5858 & 0,0001 \\
\hline & mandíbula & prono & 0,3716 & 0,0154 \\
\hline & mandíbula & sentado & 0,4740 & 0,0016 \\
\hline & mandíbula & em pé & 0,3789 & 0,0134 \\
\hline
\end{tabular}


Os resultados obtidos das maiores porcentagens de níveis em cada variável motora e oral demonstraram-se inicialmente em níveis semelhantes, as variáveis motoras elevaram seus níveis antes que as orais a partir do quinto mês até o vigésimo quarto mês. Os níveis de habilidade prono e supino elevaram-se antes da habilidade de sentar e esta antes da habilidade de ficar em pé.

O nível 1 de língua, lábios e mandíbula aos três meses com colher, apresentou-se concomitante ao nível 2 e 3 aos três e quatro meses de habilidade motora. Houve concordância entre nível 3 de língua, lábios e mandíbula em amamentação/mamadeira com nível 3 nas habilidades motoras (supino, prono e sentado) aos 4 meses.

Com 5, meses as estruturas orais com colher passaram para nível 2, simultaneamente supino e prono passaram para nível 4.

Entre 4 a 9 meses houve maior distribuição das porcentagens nos níveis de habilidades orais e motoras globais.
O nível da mandíbula aos 6 e 9 meses foi mais elevado que os de língua e lábios com colher, ocorrendo o mesmo com o copo aos 9 e 12 meses, concomitantemente aos 6 meses elevou-se o nível de supino. Aos 9 meses as maiores porcentagens das crianças encontram-se nos níveis mais elevados em supino, prono e sentado. Nota-se nessa idade porcentagens semelhantes entre a mandíbula com colher e copo e habilidade de sentado. Os níveis de habilidade de língua, lábios e mandíbula com colher igualaram aos 12 meses no nível 4, enquanto com copo somente a mandíbula atinge esse nível concomitante aos níveis mais elevados supino e prono, nível 6; sentado e em pé, nível 7 (Tabela 2).

Com 24 meses os níveis orais com copo igualaram-se no nível 4 e na mastigação atingiram nível 5, estando a maioria das crianças nos maiores níveis de habilidades motoras orais e globais (Tabela 3).

TABELA 2. Relação entre habilidade motora oral (colher, mastigação, copo) e motora global 5 a 12 meses.

\begin{tabular}{|c|c|c|c|c|}
\hline Idade & Colher & Mastigação & Copo & Motor \\
\hline 5 meses & $\begin{array}{c}\text { língua } 69 \% \text { - } 2 \\
\text { lábios } 60 \% \text { - } 2 \\
\text { mandíbula } 76 \% \text { - } 2\end{array}$ & $\cdots$ & -- & $\begin{array}{c}\text { supino } 62 \%-4 \\
\text { prono } 38 \%-4 \\
\text { sentado } 90 \%-3 \\
\text { pé } 52 \%-3\end{array}$ \\
\hline 6 meses & $\begin{array}{c}\text { língua } 55 \%-2 \\
\text { lábios } 62 \%-2 \\
\text { mandíbula.53\% }-3\end{array}$ & $\begin{array}{c}\text { língua } 94 \% \text { - } 2 \\
\text { lábios } 69 \% \text { - } 2 \\
\text { mandíbula } 76 \% \text { - } 2\end{array}$ & $\begin{array}{c}\text { língua 95\% - } 1 \\
\text { lábios } 97 \% \text { - } 1 \\
\text { mandíbula 90\% - } 1\end{array}$ & $\begin{array}{c}\text { supino } 52 \%-5 \\
\text { prono } 40 \%-6 \\
\text { sentado } 57 \%-4 \\
\text { pé } 88 \%-3\end{array}$ \\
\hline 9 meses & $\begin{array}{c}\text { língua } 61 \%-3 \\
\text { lábios } 62 \%-3 \\
\text { mandíbula.50\% }-4\end{array}$ & $\begin{array}{c}\text { língua } 85 \% \text { - } 3 \\
\text { lábios } 74 \% \text { - } 3 \\
\text { mandíbula } 86 \% \text { - } 3\end{array}$ & $\begin{array}{c}\text { língua } 74 \%-2 \\
\text { lábios } 69 \%-2 \\
\text { mandíbula } 53 \%-3\end{array}$ & $\begin{array}{c}\text { supino } 90 \%-6 \\
\text { prono } 79 \%-6 \\
\text { sentado } 48 \%-7 \\
\text { pé } 62 \%-4\end{array}$ \\
\hline 12 meses & $\begin{array}{c}\text { língua } 86 \% \text { - } 4 \\
\text { lábios } 81 \% \text { - } 4 \\
\text { mandíbula } 100 \% \text { - } 4\end{array}$ & $\begin{array}{c}\text { língua } 86 \% \text { - } 4 \\
\text { lábios } 81 \% \text { - } 4 \\
\text { mandíbula } 95 \% \text { - } 4\end{array}$ & $\begin{array}{c}\text { língua } 72 \%-3 \\
\text { lábios } 69 \%-3 \\
\text { mandíbula } 53 \%-4\end{array}$ & $\begin{array}{l}\text { supino } 100 \%-6 \\
\text { prono } 100 \%-6 \\
\text { sentado } 100 \%-7 \\
\text { pé } 69 \%-7\end{array}$ \\
\hline
\end{tabular}

TABELA 3. Relação entre habilidade motora oral (colher, mastigação, copo) e motora global 12 a 24 meses.

\begin{tabular}{c|c|c|c}
\hline Idade & Mastigação & Copo & Motor \\
\hline & língua $100 \%-5$ & língua $96 \%-4$ & supino $100 \%-6$ \\
24 meses & lábios $100 \%-5$ & lábios $95 \%-4$ & $100 \%-6$ \\
& mandíbula $100 \%-5$ & mandíbula $100 \%-4$ & sentado $100 \%-7$ \\
& & & $100 \%-7$ \\
\hline
\end{tabular}




\section{Discussão}

Estudo observacional, documentando mudanças simultâneas que ocorrem com habilidades motoras globais e orais nos dois primeiros anos de vida. Obteve-se associação entre essas habilidades na maioria dos momentos avaliados. Nas idades intermediárias (4 e 9 meses) houve mescla dos níveis apresentados caracterizando variabilidade da aquisição do desenvolvimento motor, descrita em crianças normais no primeiro e no segundo ano ${ }^{(27-28)}$.

Aos 3 e 4 meses, as habilidades para amamentação/mamadeira apresentaram-se no nível mais alto enquanto na mesma idade demonstravam competência oral com colher em nível 1, notandose inicialmente, presença de padrões orais semelhantes aos de sucção em mamadeira caracterizando desempenho oral na fase inicial dessa habilidade que mescla sucção com "suckling" (28,32-34). Nessa idade observou-se porcentagem de níveis semelhantes em supino, prono e sentado, com aumento na habilidade de sentar do nível 3 de $62 \%$ no $3^{\circ}$ mês para $96 \%$ no $4^{\circ}$ mês, demonstrando evolução na aquisição de componentes motores que habilitarão a criança sustentar a cabeça e o tronco. No desenvolvimento normal o controle da cabeça e estabilidade do tronco fornece a base motora para a função motora fina das mãos e boca e os movimentos motores orais para alimentação ocorrem após a cabeça e o tronco terem adquirido estabilidade, simetria e alinhamento ${ }^{(2,9,24,29)}$.

No $5^{\circ}$ mês manteve-se a relação entre a habilidade motora global e oral, as estruturas orais com colher elevaram seus níveis concomitante a elevação dos níveis de habilidades motoras, confirmando alguns estudos ${ }^{(5-6)}$.

No $6^{\circ}$ mês somente a mandíbula elevou-se para nível 3, mantendo-se estável para a entrada do alimento na boca, paralelamente elevaram-se os níveis em supino, prono e sentado. O movimento do lábio superior para limpar a colher durante a alimentação inicia-se aos 6 meses e depende da estabilidade da mandíbula e do tronco ${ }^{(2,26)}$. Nesta idade as estruturas orais na mastigação e copo encontravam-se em níveis inferiores, indicando a imaturidade motora oral.

Com 9 meses com colher as estruturas orais elevaram novamente seus níveis, entretanto a mandíbula apresentou características motoras mais elaboradas que língua e lábios, demonstrando sua importância no desempenho oral, devido ao fato de ser a estrutura oral mais próxima da cintura escapular e do tronco ${ }^{(24,26)}$, oferecendo estabilidade para as estruturas orais trabalharem ${ }^{(24)}$. Nessa idade demonstrou-se que os níveis das habilidades motoras globais elevaram-se, e a maior porcentagem das crianças apresentava domínio nas posições supino e prono, elevando o nível na habilidade de sentado.

Aos 12 meses com colher a língua, lábios e mandíbula atingiram seus níveis mais altos, simultaneamente as habilidades motoras apresentavam níveis mais elevados em supino, prono, sentado e em pé.

Com copo aos 9 e 12 meses observou-se comportamentos orais semelhantes aos que ocorreram com colher. A mandíbula apresentou nível superior as demais estruturas, sinalizando início da maturação progressiva para realização da função, no entanto a presença de padrão de sucção, engasgos ${ }^{(2)}$, abertura exagerada da boca, mordida na borda do copo, entre outros decorreram da pouca estabilidade da mandíbula, mesmo estando presentes à sustentação da cabeça ${ }^{(2,26,29)}$. Lábios e língua atingiram o mesmo nível de mandíbula aos 24 meses, provavelmente devido a interdependência entre a aquisição de estabilidade da mandíbula e maior habilidade de lábios e língua, dado apontado pela literatura ${ }^{(26)}$.

$\mathrm{Na}$ mastigação, as três estruturas elevaram seus níveis simultaneamente. Aos 6 meses observou-se movimento sem dissociação entre as estruturas sendo a dissociação observada aos 9 meses quando as estruturas orais elevaram seus níveis enquanto que em supino, prono e sentado encontravam-se em seus níveis mais elevados ${ }^{(6)}$.

Os movimentos diagonais da mandíbula durante a mastigação ocorreram aos 12 meses, e aos 24 meses, observou-se movimentos rotatórios e controlados e lateralização da língua ${ }^{(2,26)}$.

No desenvolvimento das habilidades motoras observou-se seqüência no desenvolvimento do controle postural em supino e prono, obtendo-se resultados semelhantes aos descritos por Green et al. ${ }^{(12)}$ e aos descritos em estudo com crianças até três meses por Gaetan et al. ${ }^{(30)}$. O domínio na capacidade de sentar foi pré-requisito para o desenvolvimento adequado do controle oral e para aquisições motoras orais ${ }^{(5-6,9-10,24)}$.

Esse estudo enfatiza a influência entre a postura corporal sobre a estrutura oral e vice-versa na alimentação nas desordens sensório-motoras $(11,13,15)$ sendo uma observação detalhada longitudinal em crianças normais. 


\section{Conclusão}

Podemos afirmar em conclusão que as crianças apresentaram habilidades motoras orais dentro dos padrões considerados normais, que essas habilidades desenvolveram-se concomitantemente as habilidades motoras globais, havendo associação entre ambas.

\section{Referências Bibliográficas}

1. Morris SE. The normal acquisition of oral feeding skills: implications for assessment and treatment. Central Islip: NY: Therapeutic Media, 1982.

2. Stevenson RD, Allaire JH. The development of normal feeding and swallowing. Pediatr. Clin. North Am. 1991;38:1439-53

3. Diament A, Cypel S. Neurologia infantil. São Paulo: Atheneu., 2005. p.3-4,11-12.

4. Arqueles PP, Almirall CB, Metayer M, Sanclemente MP. A fonoaudiologia na paralisia cerebral. São Paulo: Santos Livraria Editora, 2001. p.1-10.

5. Morris SE. A profile of the development of oral motor skills in early infancy - birth to 12 months, (manual). Faber, VA; 1991.

6. Carruth BR, Skinner JD. Feeding behaviors and other motor development in healthy children (2 - 24 moths). American college of Nutrition Journal. 2002;21:88-96.

7. Marquezan IQ. Atuação Fonoaudiológica nas Funções Orofaciais. in: Avaliação e Tratamento em Fonoaudiologia em Pediatria. São Paulo: Sarvier; 2003. p. 3.

8. Sanches MTC. Manejo clínico das disfunções orais na amamentação. J. Pediatr (Rio J). 2004;(80 Supl 5):S15562 .

9. Wolf LS, Glass RP. Feeding and swallowing disorders in infancy: assessment and management. Texas: Therapy skill Builders; 1992.

10. Redstone F, West JF. The importance postural control for feeding. Pediatric. Nussing Journal. 2004;30(2):97-100.

11 - Gaetan MG, Ribeiro MVLM. Recém Nascido pré-termo. In: Ribeiro MVLM, Gonçalves VMG. Neurologia do desenvolvimento. Rio de Janeiro: Revinter; 2006. cap. 14.

12. Green EM, Mulcahy CM, Pountney TE. An investigation into the development of early postural control. Dev. Med. Child Neurol. 1995;37:437-48.

13. Gisel GE, Schuwartz S, Petryk A, Clarke D, Haberfellner $\mathrm{H}$. Whole body mobility after one year of intraoral appliance therapy in children which cerebral palsy and moderate eating impairment. Dysphagia. 2000;15(4):226-35.

14. West JF, Redstone F. Aligment during feeding and swallowing does it matter? A reviw. Perceptual and Motor Skills. 2004 feb;98(1):348-58.

15. Val DC, Limongi SCO, Fabiano FC, Silva KCL. Sistem estomatognático e postura corporal na criança com alterações sensório-motoras. Pró-Fono Revista de Atualização Científica, Barueri (SP). 2005;17(3):345-54.
A limitação do estudo é o fato de ser observacional, e o ponto positivo foi o elevado grau de concordância entre os examinadores.

Pesquisas futuras com medidas de tempo desde a entrada do alimento na boca até sua deglutição poderão fornecer dados quantitativos normativos nas diferentes idades em crianças normais.

16. Dusick A. Investigacion and management of dysphagia. Semin. Pediatr. Neurol. 2003;10(4):255-64.

17. Haberfellner H. Ismar: an autitherapeutic device assisting patients from drooling to articulated seepch. Pediatr Rehabil. 2005;8(4):248-62.

18. Gisel GE. Effect of food texture on the development of chewing of children between six months and two years of age. Dev. Med. Child Neurol. 1991;33:69-79.

19. Ramsay M, Gisel EG. Neonatal sucking and maternal feeding practices. Dev. Med. Child Neurol. 1996;38:34-7.

20. Tamura Y, Matusushita S, Shinoda K, Yoshida S. Development of perioral muscle activity during suckling in infants: a cross-sectional and follow-up study. Dev. Med. Child Neurol. 1998;40:344-8.

21. Quareshi MA, Vice FL, Taciak VL, Bosna JF, Gewolh IH. Changes in rhythimic suckle feeding patterns in term infants in the first month of live. Dev. Med. Child Neurol. 2002;44:34-9.

22. Neiva FCB, Cottoni DM, Ramos JLA, Issler H. Desmame precoce: implicações para o desenvolvimento motor oral. J. Pediatr (Rio J). 2003;79:7-12.

23. Lubchenco LO, Hansman C, Boyd E. Intrauterine Growth as estimated from liveborn birth-weigth data at 24 to 42 weeks of gestacion. Pediatrics. 1963;32:792-800.

24. BLY L. Motor skills in the first years. Tucson, Arizona, USA. Therapy Builders Skills, 1994.

25. Nardi MJO, Porto MRS. Problemas ortopédicos na criança. In: Kudo AM, Marcodes M, Lins L, Moriyama LT, Guimarães ML, Juliani RCTP, Pierri SA. Fisioterapia fonoaudiologia e terapia ocupacional em pediatria, 2a. ed. São Paulo: Sarvier; 1994. p. 37-8.

26. Morris SE, Klein MD. Pré-feeding skills. Tucson, Therapy Skill Builder, 1987.

27. Touwen BCL. A study on the development of some motor phenomena in infant. Dev. Med. Child Neurol. 1971;13:435-46.

28. Dargassies A. Neurodevelopmental symptoms during the first year of life. Dev. Med. Child Neurol. 1972;14:23546.

29. Hernandez AM. O neonato: In Hernandez AM (Org.). Atuação fonoaudiológica com o sistema estomatognático e a função de alimentação. São José dos Campos, São Paulo: Pulso; 2003. p. 47-61.

30. Gaetan MG, Ribeiro MVLM. Developmental study of early posture control in preterm and fullterm infants. Arq. Neuro-Psiquiatr. $2002 \mathrm{dec} ; 60(4)$. 